ORIGINAL ARTICLE

\title{
Differential prognostic impact of hypoxia induced and diffuse HIF- $1 \alpha$ expression in invasive breast cancer
}

\author{
M M Vleugel, A E Greijer, A Shvarts, P van der Groep, M van Berkel, Y Aarbodem, H van \\ Tinteren, A L Harris, P J van Diest, E van der Wall
}

J Clin Pathol 2005;58:172-177. doi: 10.1136/icp.2004.019885

See end of article for authors' affiliations

Correspondence to:

Correspondence to:
Professor P J van Diest Department of Pathology, University Medical Centre Utrecht, PO Box 85500, 3508 GA Utrecht, The Netherlands:

P.J.vanDiest@lab.azu.nl

Accepted for publication 24 June 2004
Background: Intratumorous hypoxia triggers a broad cellular response mediated by the transcription factor hypoxia inducible factor 1 (HIF-1). HIF-1 $\alpha$ concentrations increase during breast carcinogenesis, and are associated with poor prognosis. An earlier study noted two HIF- $1 \alpha$ overexpression patterns: diffuse scattered throughout the tissue and confined to perinecrotic cells.

Aims: To investigate the prognostic impact of these different HIF-1 $\alpha$ overexpression patterns in relation to its downstream effectors carbonic anhydrase (CA) IX and glucose transporter 1 (GLUT-1).

Methods: HIF- $1 \alpha$, CA IX, and GLUT-1 expression was studied by immunohistochemistry, including double staining for CA IX and HIF-1 $\alpha$. Clinical data included disease free survival, lymph node status, and tumour size.

Results: HIF- $1 \alpha$ overexpression (44\% of cases) had a perinecrotic $(13.5 \%$ ) or diffuse staining pattern (30.5\%). CA IX expression was detectable in $12.5 \%$ of breast cancers, whereas GLUT-1 expression was seen in $29 \%$, with both showing perinecrotic membrane staining. Perinecrotic HIF-1 $\alpha$ overexpression was highly associated with CA IX and GLUT-1 overexpression, and double staining for HIF-1 $\alpha$ and CA IX showed strong expression in the same cells. Diffusely overexpressed HIF- $1 \alpha$ was not associated with CA IX or GLUT-1 expression. Patients with diffuse HIF-1 $\alpha$ staining had a significantly better prognosis than patients with perinecrotically overexpressed HIF- $1 \alpha$.

Conclusions: Different regulation pathways of HIF-1 $1 \alpha$ overexpression exist in breast cancer: (1) hypoxia induced, perinecrotic HIF-1 $\alpha$ overexpression with strong expression of hypoxia associated genes (CA IX and GLUT-1), which is associated with a poor prognosis; and (2) diffuse HIF-1 $\alpha$ overexpression lacking major hypoxia associated downstream effects, resulting in a more favourable prognosis.
$\mathrm{H}$ ypoxia is one of the hallmarks of cancer. The presence of hypoxia has been demonstrated in different types of solid tumours, including breast cancer. ${ }^{1}$ Intratumorous hypoxia is caused by the lack of functional blood vessels in proliferating tumour tissue, resulting in low intratumorous oxygen concentrations. If hypoxia is severe or prolonged, cell death occurs. Malignant cells can undergo genetic and adaptive changes that allow them to escape from dying of oxygen deprivation. These changes are associated with a more aggressive malignant phenotype, and resistance to chemotherapy and radiotherapy, resulting in a poor prognosis. $^{23}$

\section{"Malignant cells can undergo genetic and adaptive changes that allow them to escape from dying of oxygen deprivation"}

The broad cellular response caused by hypoxia is mediated by the action of the transcription factor hypoxia inducible factor 1 (HIF-1). HIF-l binds to the hypoxia response element in promoters of various target genes. Hypoxia inducible genes regulate several crucial biological processes, including apoptosis, migration (metastasis), cell proliferation, angiogenesis, and glycolytic metabolism. ${ }^{4}$ Changes in the expression patterns of these genes counteract hypoxia and increase oxygenation, whereas others affect the cellular adaptation to decreased oxygen concentrations or mediate cell death signalling pathways. ${ }^{56}$

The HIF-l protein is a heterodimer consisting of two subunits, HIF- $1 \alpha$ and HIF- $1 \beta .^{7}$ HIF- $1 \beta$ is constitutively expressed and will bind to HIF- $1 \alpha$ when concentrations of
HIF- $l \alpha$ are high. Under normoxic conditions, hydroxylation of HIF- $1 \alpha$ allows the von Hippel Lindau E3 ubiquitin ligase complex to bind to HIF- $1 \alpha$, which is then degraded by the proteasome. ${ }^{8}$ During hypoxia, stabilisation of HIF- $1 \alpha$ occurs through inhibition of 4-prolyl hydroxylase activity, an enzyme that requires oxygen to be functional. ${ }^{9}$ Upon stabilisation, HIF- $1 \alpha$ protein is transported into the nucleus where it heterodimerises with HIF-1 $\beta$, forming the active HIF-1 transcription complex. Oxygen independent HIF-l $\alpha$ expression may occur by genetic alterations, such as loss of tumour suppressor genes or activation of oncogenes. ${ }^{10}$

Increased concentrations of HIF-l $\alpha$ are found in many solid human cancers. ${ }^{11}$ In breast cancer, increased concentrations of HIF-l $\alpha$ were associated with increased proliferation and poor differentiation, indicating the association of HIF- $1 \alpha$ expression with a more aggressive phenotype. ${ }^{12}$ Several studies have reported the prognostic relevance of HIF- $1 \alpha$ overexpression in human cancer..$^{13}$ In breast cancer, increased concentrations of HIF-l $\alpha$ are independently associated with shortened survival in lymph node negative patients. ${ }^{15}$ In these tissue studies, it was noted that HIF-l $\alpha$ overexpression may be diffuse throughout the tumour or may be seen in perinecrotic tumour cells. Whether these different patterns have different cell biological and prognostic implications is yet unknown. One crucial aspect of this is whether there is a differential activation of HIF-1 downstream target genes.

Abbreviations: CA IX, carbonic anhydrase IX; DFS, disease free survival; GLUT-1, glucose transporter 1; HIF-1, hypoxia inducible factor 1; HRE, hypoxia response element; VEGF, vascular endothelial growth factor 
Table 1 Association of HIF- $1 \alpha$ expression with clinicopathological features in patients with invasive breast cancer $(n=200)$

\begin{tabular}{|c|c|c|c|c|}
\hline & $\mathbf{n}$ & $\begin{array}{l}\text { HIF- } 1 \alpha \\
<1 \%\end{array}$ & $\begin{array}{l}\text { HIF- } 1 \alpha \\
>1 \%\end{array}$ & $p$ Value $\left(\chi^{2}\right)$ \\
\hline Total & 200 & 112 & 88 & \\
\hline \multicolumn{5}{|c|}{ Histological type } \\
\hline Ductal & 144 & 76 & 68 & \\
\hline Lobular & 30 & 22 & 8 & \\
\hline Tubular & 11 & 9 & 2 & \\
\hline Papillary & 2 & 1 & 1 & \\
\hline Mucinous & 4 & 1 & 3 & \\
\hline Medullary & 4 & 0 & 4 & \\
\hline Apocrine & 2 & 0 & 2 & \\
\hline Cribriform & 3 & 3 & 0 & $N A^{*}$ \\
\hline \multicolumn{5}{|c|}{ Histological grade } \\
\hline 1 & 61 & 46 & 15 & \\
\hline ॥ & 78 & 48 & 30 & \\
\hline III & 61 & 18 & 43 & $<0.001$ \\
\hline \multicolumn{5}{|l|}{ Tumour size } \\
\hline $0-2 \mathrm{~cm}$ & 97 & 61 & 36 & \\
\hline $2-5 \mathrm{~cm}$ & 89 & 42 & 47 & \\
\hline$>5 \mathrm{~cm}$ & 14 & 9 & 5 & 0.080 \\
\hline \multicolumn{5}{|c|}{ Lymph node status } \\
\hline Negative & 115 & 69 & 46 & \\
\hline Positive & 72 & 35 & 37 & 0.127 \\
\hline \multicolumn{5}{|l|}{ Necrosis } \\
\hline Negative & 162 & 101 & 61 & \\
\hline Positive & 38 & 11 & 27 & $<0.001$ \\
\hline \multicolumn{5}{|l|}{ CAIX } \\
\hline Negative & 175 & 108 & 67 & \\
\hline Positive & 25 & 4 & 21 & $<0.001$ \\
\hline \multicolumn{5}{|l|}{ GLUT-1 } \\
\hline Negative & 143 & 97 & 46 & \\
\hline Positive & 57 & 15 & 42 & $<0.001$ \\
\hline
\end{tabular}

*Lobular breast carcinomas showed significantly less frequent HIF- $1 \alpha$ expression than ductal cancers $(p=0.03)$.

CA IX, carbonic anhydrase IX; GLUT-1, glucose transporter 1; HIF-1 $\alpha$, hypoxia inducible factor $1 \alpha$; NA, not associated.

Carbonic anhydrase IX (CA IX), a member of the family of zinc metalloenzymes, is a major downstream target of HIF$1 \alpha .{ }^{16}$ Its function is to regulate intracellular and extracellular $\mathrm{pH}$ by converting $\mathrm{CO}_{2}$ to carbonic acid. Previous clinical studies revealed that CA IX has prognostic value in breast cancer, ${ }^{17}{ }^{18}$ cervical cancer, ${ }^{19}$ non-small cell lung cancer, ${ }^{20}$ and nasopharyngeal cancer. ${ }^{21}$ In these studies, significant associations were found between CA IX expression and necrosis. Because the presence of necrosis represents the result of previous and ongoing intratumorous hypoxia, ${ }^{22}{ }^{23}$ these results indicate that CA IX is an intrinsic marker of tumour hypoxia.

Glucose transporter 1 (GLUT-1) is a membrane bound protein involved in glucose transport, which is frequently overexpressed in tumours, thereby allowing increased glucose uptake of cancer cells. ${ }^{24}$ Expression of GLUT-1 was found to be regulated by hypoxia in a HIF-1 dependent way. ${ }^{25}$

We hypothesised that the perinecrotic and diffuse HIF-l $\alpha$ expression patterns may have different cell biological and clinical implications. Therefore, we investigated these HIF- $1 \alpha$ expression patterns in a large group of patients with invasive breast cancer with longterm follow up, in relation to its downstream targets, CA IX and GLUT-1.

\section{MATERIALS AND METHODS}

Paraffin wax embedded material was still available from 200 patients who formed part of a previously extensively described group of patients with invasive breast cancer. ${ }^{26}$ Anonymous use of leftover tumour material is part of the standard treatment agreement with patients in our hospital.
All tumours had been fixed for at least 24 hours in neutral buffered $4 \%$ formaldehyde, were classified according to the World Health Organisation, and were graded according to a modification of Bloom and Richardson's method. ${ }^{27}$ Histological classification revealed 144 ductal, 30 lobular, 11 tubular, two papillary, four mucinous, four medullary, two apocrine, and three cribriform cancers. The mean tumour diameter was $2.2 \mathrm{~cm}$, ranging from 0.3 to $10.0 \mathrm{~cm}$. The group included 72 lymph node positive and 115 lymph node negative patients, whereas for 13 patients lymph node status was unknown. None of the patients received preoperative chemotherapy, hormonal treatment, or radiotherapy. Adjuvant systemic treatment (chemotherapy for premenopausal and tamoxifen for postmenopausal patients) was given according to established guidelines. Oestrogen receptor status had been determined routinely by immunohistochemistry.

\section{Immunohistochemistry}

Immunohistochemistry was performed on $4 \mu \mathrm{m}$ thick serial sections derived from formaldehyde fixed, paraffin wax embedded tumour tissue blocks. All sections were dewaxed and rehydrated.

HIF- $1 \alpha$ staining was performed as described previously. ${ }^{12}$ In short, antigen retrieval was performed ( 45 minutes at $96^{\circ} \mathrm{C}$ ) in target retrieval solution (Dako, Glostrup, Denmark). The primary mouse antibody (anti-HIF- $1 \alpha ; 1 / 500$ dilution; BD Transduction Laboratories, Lexington, Kentucky, USA) was incubated for 30 minutes at $20^{\circ} \mathrm{C}$. The catalysed signal amplification system (Dako) was used to detect HIF-l $\alpha$ staining.

For CA IX staining, sections were incubated without antigen retrieval with mouse anti-CA IX (MN 75; 1/50 dilution; kindly provided by Dr S Pastorekova, Institute of Virology, Academy of Science, Bratislava, Slovakia) for 30 minutes at $20^{\circ} \mathrm{C}$ and subsequently developed with an avidinbiotin-peroxidase complex method (Envision system peroxidase mouse; Dako). All sections were developed using diaminobenzidine, and subsequently counterstained with haematoxylin.

For GLUT-1 staining, sections were incubated without antigen retrieval with a rabbit polyclonal anti-GLUT-1 antibody (clone A 3536; Dako) and subsequently developed with a standard avidin-biotin-peroxidase complex method (biotinlyated goat antirabbit antibody; Dako; streptavidin peroxidase system; Dako) using an autostainer (Autostainer 480-2D; LabVision, Freemont, California, USA).

Double staining for CA IX and HIF- $1 \alpha$ was achieved by first performing HIF- $1 \alpha$ single staining, as described above. After diaminobenzidine development, sections were incubated with mouse anti-CA IX antibody ( $1 / 50$ dilution) and the Power Vision System antimouse $\mathrm{AP}^{\circledR}$ (Dako), and subsequently stained with Fast Blue BB (Sigma, St Louis, Missouri, USA). Before the slides were mounted, all sections were dehydrated in alcohol and xylene.

\section{Quantification}

The percentages of nuclei positive for HIF-l $\alpha$ were scored blindly by two observers (PvD and MMV), only counting homogeneously and darkly stained nuclei as positive. For HIF- $1 \alpha$, a percentage of $\geqslant 1 \%$ positive nuclei was considered positive, as described previously. ${ }^{12}{ }^{15}$ Reanalysis of different HIF- $1 \alpha$ thresholds for the present data set again made it clear that this was the optimal prognostic cutoff value. For CA IX and GLUT-1, staining was scored as positive or negative, with any membranous staining around tumour cells classed as positive. Using a magnification of $\times 200$, the presence of any necrosis on haematoxylin and eosin stained sections was scored as positive. 

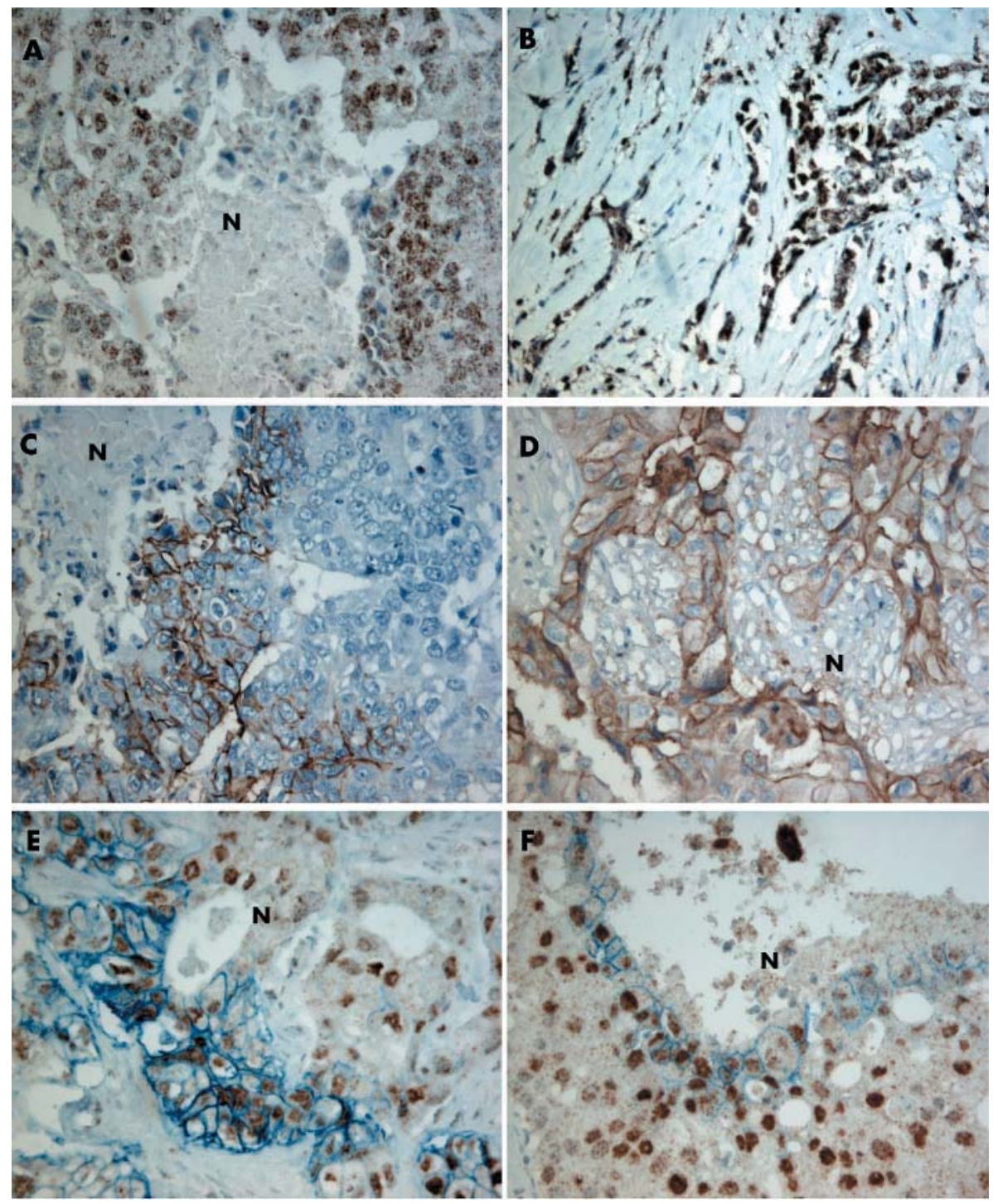

Figure 1 Immunohistochemical analysis of hypoxia inducible factor $1 \alpha$ (HIF-1 $\alpha$ ) and carbonic anhydrase IX (CA IX) in human breast cancer. (A) HIF$1 \alpha$ nuclear expression around necrotic (N) areas. (B) Diffuse nuclear HIF-1 $\alpha$ expression, not related to necrosis. (C) CA IX membrane expression around necrotic areas. (D) Glucose transporter 1 (GLUT-1) membrane expression around necrotic areas. (E, F) Double staining revealing strong staining of HIF- $1 \alpha$ and CA IX around necrotic areas (original magnification, $\times 200$ ).

Table 2 Associations between HIF-1 $\alpha$ staining pattern, grade, and expression of CA IX and GLUT-1 for HIF-1 $\alpha$ positive breast cancer cases $(n=88)$

\begin{tabular}{|c|c|c|c|c|}
\hline & \multirow[b]{2}{*}{$\mathbf{n}$} & \multicolumn{2}{|c|}{ HIF- $1 \alpha$ staining pattern* } & \multirow[b]{2}{*}{ p Value $\left(\chi^{2}\right)$} \\
\hline & & Diffuse & Necrotic & \\
\hline \multicolumn{5}{|l|}{ Grade } \\
\hline I & 15 & 13 & 2 & \\
\hline II & 30 & 25 & 5 & \\
\hline III & 43 & 23 & 20 & 0.007 \\
\hline \multicolumn{5}{|l|}{ CA IX } \\
\hline Negative & 67 & 55 & 12 & \\
\hline Positive & 21 & 6 & 15 & $<0.001$ \\
\hline \multicolumn{5}{|l|}{ GLUT-1 } \\
\hline Negative & 46 & 41 & 5 & \\
\hline Positive & 42 & 20 & 22 & $<0.001$ \\
\hline \multicolumn{5}{|c|}{$\begin{array}{l}\text { *One case, which was grouped with the perinecrotic cases, showed both } \\
\text { diffuse and perinecrotic expression. } \\
\text { CA IX, carbonic anhydrase IX; GLUT-1, glucose transporter } 1 ; \text { HIF- } 1 \alpha \text {, } \\
\text { hypoxia inducible factor } 1 \alpha \text {. }\end{array}$} \\
\hline
\end{tabular}

\section{Statistical and survival analysis}

The $\chi^{2}$ test (SPSS for Windows version 9.01; 1999 SPSS Inc, Chicago, Illinois, USA) was used to test the correlations between HIF-1 $\alpha$, CA IX, GLUT-1, and the presence of necrosis. Two sided $\mathrm{p}$ values $<0.05$ were considered significant.

For survival analysis, clinical data were available from 166 patients. The endpoint of analysis was recurrence, defined as the first reappearance of breast cancer at any site or death (disease free survival (DFS) analysis). Survival estimates and curves were calculated using the Kaplan-Meier technique and differences in time to recurrence were tested by means of a log rank test.

\section{RESULTS}

HIF- $1 \alpha$ expression was detectable in 88 of $200(44 \%)$ patients with breast cancer. Two staining patterns were observed: predominantly around necrotic areas for 27 cases (fig lA) or diffusely throughout the tumour for 61 cases (fig 1B). Nuclear HIF- $1 \alpha$ expression was occasionally demonstrated in normal tissue surrounding the tumour. Cytoplasmic 


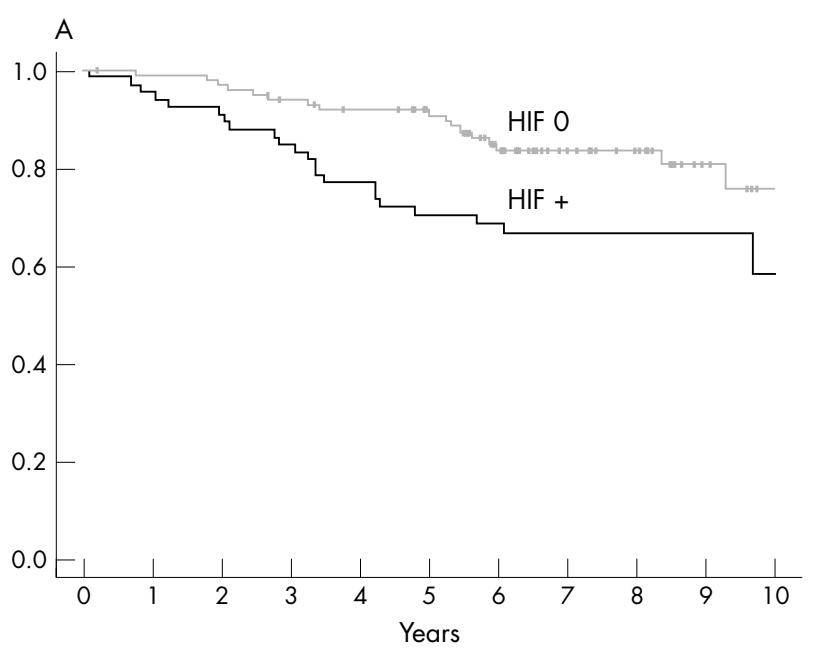

Forty one HIF- $1 \alpha$ positive cases showed no CA IX or GLUT1 expression. Thirty nine of 41 of these cases showed a diffuse HIF-1 staining pattern. Sixteen HIF- $1 \alpha$ positive cases showed double positivity for CA IX and GLUT-1. From these double positives, 12 of 16 showed a perinecrotic staining pattern.

In univariate survival analysis, a significant negative relation was found between HIF- $1 \alpha$ expression and DFS $(\mathrm{p}=0.01$; table 3 ; fig $2 \mathrm{~A}, \mathrm{~B})$, with a relative 2.2 fold increased risk for recurrences compared with the HIF-l $\alpha$ negative group. A separate analysis according to type of HIF$1 \alpha$ expression showed that perinecrotic HIF- $1 \alpha$ expression was associated with a worse prognosis (relative risk, 3.1) than diffuse HIF-1 $\alpha$ expression (relative risk, 2.0; table 3 ).

\section{DISCUSSION}

In a previous study, we noted two HIF-l $\alpha$ overexpression patterns: diffuse throughout the tumour and restricted to perinecrotic tumour cells. ${ }^{12}$ This was confirmed in our present study, in which we performed immunohistochemical and survival analyses in a group of 200 patients with breast cancer. We used whole tissue sections because there is often pronounced intratumour heterogeneity for HIF-l $\alpha$ staining because of focal perinecrotic staining, which is clinically relevant. Data derived from a tissue array study would probably be less reliable with regard to associations between HIF- $1 \alpha$ and prognosis. Indeed, a recent study found only $5 \%$ of breast cancers to be HIF- $1 \alpha$ positive on tissue microarray. ${ }^{28}$

Nuclear HIF- $1 \alpha$ overexpression was seen in $44 \%$ of breast cancer cases by single immunohistochemical staining, mainly in ductal, medullary, and apocrine types, and very rarely in lobular, tubular, and cribriform cancers. Strong CA IX and GLUT-1 membrane staining was seen in $13 \%$ and $29 \%$ of cancers, respectively, and was mainly confined to areas around necrosis. Previous single staining studies have reported similar expression levels for HIF- $1 \alpha$ and CA IX as individual markers in invasive breast cancer, ${ }^{17}{ }^{29}$ non-small cell lung cancer, ${ }^{19}$ and nasopharyngeal carcinoma. ${ }^{17}$

Perinecrotic HIF- $1 \alpha$ expression was seen in 27 of 88 HIF- $1 \alpha$ positive cancers and was significantly associated with the expression of CA IX and GLUT-1. Tomes et al previously showed HIF- $1 \alpha$ expression around necrosis with strong expression of its downstream genes CA IX, GLUT-1, and vascular endothelial growth factor (VEGF). ${ }^{30}$ However, our present study is the first to confirm the coexpression of HIF$1 \alpha$ and CA IX in the same cells by double staining experiments. In addition to the perinecrotic staining observed by Tomes et al, we have demonstrated HIF- $1 \alpha$ positivity with a diffuse staining pattern. Interestingly, these diffuse HIF- $1 \alpha$ positive cases (61 of 88) did not show coexpression of CA IX or GLUT-1 expression as the perinecrotic HIF- $1 \alpha$ positive cases did.

GLUT-1 expression is induced by hypoxia in a HIF-1 dependent way. ${ }^{25}$ However, we found that GLUT-1 was also sometimes overexpressed in the absence of HIF-l $\alpha$ overexpression (15 of 200 cases). In these cases, GLUT-1 overexpression might be induced by other factors, such as growth factors, mitogens, and activated oncogenes. ${ }^{25}$ In contrast, only four breast cancer cases showed CA IX expression in the absence of HIF- $1 \alpha$ expression. Because CA IX expression is induced at a reduced oxygen concentration, but above that necessary for HIF- $1 \alpha$ stabilisation, it is possible that CA IX expression may also be induced independently of HIF- $1 \alpha$ activation. ${ }^{31}$ Furthermore, CA IX expression is seen not only in hypoxic tumour regions, but also in those regions previously affected by hypoxia. ${ }^{32}$ Nevertheless, our results showed that, in contrast to GLUT-1, CA IX expression is more tightly regulated by hypoxia. Therefore, CA IX might be a useful marker of hypoxic areas in tumours. However, this is not an absolute relation, because

expression of HIF- $1 \alpha$ and CA IX in perinecrotic regions was confirmed by double staining (fig lE, F). 
Table 3 Disease free survival analysis of invasive breast cancer patients $(n=166$ ) according to HIF- $1 \alpha$ positivity and staining pattern

\begin{tabular}{llrllll}
\hline & Cutoff & $\mathbf{n}$ & Recurrence & p Value* & HR & 95\% Cl \\
\hline HIF-1 $\alpha$ & $\leqslant 1 \%$ & 100 & $17 \%$ & & 1.00 & 1.18 to 4.202 \\
Staining pattern & $>1 \%$ & 66 & $33 \%$ & 0.01 & 2.23 & \\
& Diffuse & 51 & $31 \%$ & 0.04 & 2.03 & 1.025 to 4.019 \\
& Necrotic & 15 & $40 \%$ & 0.02 & 3.05 & 1.200 to 7.741 \\
\hline
\end{tabular}

*Log rank test.

$\mathrm{Cl}$, confidence interval; HIF- $1 \alpha$, hypoxia inducible factor $1 \alpha$; HR, hazard ratio.

11 of 38 breast cancers with necrosis showed absence of both HIF- $1 \alpha$ and CA IX expression. In these cases, factors other than hypoxia alone may contribute to the development of necrosis, such as metabolic or vascular mechanisms. ${ }^{33}$

\begin{abstract}
"Both groups have a worse disease free survival prognosis compared with patients with $\mathrm{HIF}-1 \alpha$ negative breast cancer, indicating the potential for HIF-1 as a therapeutic target $^{\prime \prime}$
\end{abstract}

Because many diffuse HIF- $1 \alpha$ positive cases (39 of 61) showed total absence of CA IX and GLUT-1 expression, this may point to a lack of activation of HIF- 1 target genes. These variations in transcriptional activation may represent differential tumour behaviour. Therefore, we investigated the differential prognostic impact of these different staining patterns. Using (DFS) analysis, HIF- $1 \alpha$ expression was found to be a predictor of poor prognosis. These results are in agreement with our earlier results in another breast cancer patient group, ${ }^{15}$ and the results of other groups. ${ }^{13}$ Our results showed that in breast cancer, perinecrotic HIF-l $\alpha$ overexpression was associated with a worse prognosis than diffuse HIF- $1 \alpha$ overexpression, underlying the clinical importance of the presence of downstream HIF-l $\alpha$ effects. The prognostic impact of diffuse and perinecrotic HIF- $1 \alpha$ expression patterns was previously investigated in oropharyngeal cancer. In that study, the staining pattern (focal versus diffuse) had no significant influence on local tumour control or patient survival. ${ }^{34}$ Thus, our study is the first to show a differential prognostic impact of the HIF- $1 \alpha$ staining pattern in breast cancer.

HIF- $1 \alpha$ expression away from necrosis might be caused by mechanisms other than hypoxia. Cytokines and other signalling molecules stimulate HIF- $1 \alpha$ synthesis by acting on the phosphatidylinositol $3^{\prime}$-kinase or mitogen activated protein kinase pathways involved in HIF- $1 \alpha$ protein synthesis. Furthermore, HIF-l $\alpha$ expression or activity is increased in response to genetic alterations. For example, loss of function mutations in tumour suppressor genes, such as p53

Take home messages

- Although, in general, hypoxia inducible factor $1 \alpha$ (HIF$1 \alpha)$ concentrations increase during breast carcinogenesis and are associated with poor prognosis, different regulation pathways of HIF- $1 \alpha$ overexpression exist

- The first, hypoxia induced perinecrotic HIF-l $\alpha$ overexpression with strong expression of hypoxia associated genes (CA IX and GLUT-1), is associated with a poor prognosis

- The second, diffuse HIF-1 $\alpha$ overexpression lacking major hypoxia associated downstream effects, results in a more favourable prognosis and von Hippel-Lindau, influence the concentrations and functions of HIF-1. The expression of oncogenes, such as $\mathrm{v}$-Src and H-ras, has also been implicated in HIF-l $\alpha$ expression at normoxia. ${ }^{10} \mathrm{HIF}-1 \alpha$ overexpression at normoxia might also be caused by genetic changes in the HIF-l $\alpha$ gene. ${ }^{35}{ }^{36}$ These alternative HIF-1 regulation pathways need to be studied with regard to HIF-1 $\alpha$ expression patterns and HIF-1 transcriptional activation in vivo.

In conclusion, we found supporting evidence for the hypothesis that different upstream pathways exist for regulation of HIF- $1 \alpha$ expression, namely: (1) hypoxia related HIF- $1 \alpha$ induction and (2) normoxia related HIF- $1 \alpha$ induction. In hypoxic conditions, HIF-l $\alpha$ expression is seen perinecrotically, and induces hypoxia associated downstream target genes such as CA IX and GLUT-1, whereas under normoxic circumstances, HIF- $1 \alpha$ can be stabilised via other pathways, without activation of these downstream genes. The clinical importance of these normoxic versus hypoxic staining patterns is underlined by the relatively better prognosis of patients with diffuse HIF- $1 \alpha$ overexpression in their invasive breast cancers. However, both groups have a worse DFS prognosis compared with patients with HIF-l $\alpha$ negative breast cancer, indicating the potential for HIF-l as a therapeutic target.

\section{ACKNOWLEDGEMENTS}

Supported by the (unrestricted) First Aegon International Scholarship in Oncology. We thank Dr S Pastorekova, Institute of Virology, Slovak Academy of Sciences, Bratislava, Slovakia, for supply of the MN75 antibody.

\section{Authors' affiliations}

M M Vleugel, A E Greijer, M van Berkel, Y Aarbodem, Departments of Pathology/Medical Oncology, VU University Medical Centre, 1081 HV Amsterdam, The Netherlands

A Shvarts, P van der Groep, P J van Diest, Department of Pathology, University Medical Centre Utrecht, PO Box 85500, 3508 GA Utrecht, The Netherlands

H van Tinteren, The Netherlands Cancer Institute, 1066 CX Amsterdam, The Netherlands

A L Harris, Molecular Oncology Laboratory, Weatherall Institute of Molecular Medicine, John Radcliffe Hospital, Oxford OX3 9DS, UK E van der Wall, Division of Internal Medicine and Dermatology, University Medical Centre Utrecht, 3508 GA Utrecht, The Netherlands

\section{REFERENCES}

1 Vaupel P, Schlenger K, Knoop C, et al. Oxygenation of human tumors: evaluation of tissue oxygen distribution in breast cancers by computerized $\mathrm{O}_{2}$ tension measurements. Cancer Res 1991;51:3316-22.

2 Hockel M, Vaupel P. Tumor hypoxia: definitions and current clinical, biologic, and molecular aspects. J Natl Cancer Inst 2001;93:266-76.

3 Harris AL. Hypoxia-a key regulatory factor in tumour growth. Nat Rev Cancer 2002;2:38-47.

4 Forsythe JA, Jiang $\mathrm{BH}$, lyer NV, et al. Activation of vascular endothelial growth factor gene transcription by hypoxia-inducible factor 1. Mol Cell Biol 1996; 16:4604-13.

5 Carmeliet P, Dor Y, Herbert JM, et al. Role of HIF-1 alpha in hypoxia-mediated apoptosis, cell proliferation and tumour angiogenesis. Nature 1998;394:485-90.

6 Semenza GL. HIF-1 and mechanisms of hypoxia sensing. Curr Opin Cell Biol 2001;13:167-71 
7 Huang LE, Gu J, Schau M, et al. Regulation of hypoxia-inducible factor lalpha is mediated by an $\mathrm{O}_{2}$-dependent degradation domain via the ubiquitinproteasome pathway. Proc Natl Acad Sci U S A 1998;95:7987-92.

8 Jaakkola P, Mole DR, Tian YM, et al. Targeting of HIF-alpha to the von HippelLindau ubiquitylation complex by $\mathrm{O}_{2}$-regulated prolyl hydroxylation. Science $2001 ; 292: 468-72$.

9 Epstein AC, Gleadle JM, MCNeill LA, et al. C. elegans EGL-9 and mammalian homologs define a family of dioxygenases that regulate HIF by prolyl hydroxylation. Cell 2001;107:43-54.

10 Semenza GL. Targeting HIF-1 for cancer therapy. Nat Rev Cancer 2003;3:721-32.

11 Zhong H, De Marzo AM, Laughner E, et al. Overexpression of hypoxiainducible factor lalpha in common human cancers and their metastases. Cancer Res 1999:59:5830-5.

12 Bos R, Zhong H, Hanrahan CF, et al. Levels of hypoxia-inducible factor- 1 alpha during breast carcinogenesis. J Natl Cancer Inst 2001;93:309-14.

13 Schind M, Schoppmann SF, Samonigg H, et al. Overexpression of hypoxia inducible factor lalpha is associated with an unfavorable prognosis in lymph node-positive breast cancer. Clin Cancer Res 2002;8:1831-7.

14 Birner P, Gatterbauer B, Oberhuber G, et al. Expression of hypoxia-inducible factor-1 alpha in oligodendrogliomas: its impact on prognosis and on neoangiogenesis. Cancer 2001;92:165-71.

15 Bos R, van der GP, Greijer AE, et al. Levels of hypoxia-inducible factor-1 alpho independently predict prognosis in patients with lymph node negative breast carcinoma. Cancer 2003;97:1573-81.

16 Wykoff CC, Beasley NJ, Watson PH, et al. Hypoxia-inducible expression of tumor-associated carbonic anhydrases. Cancer Res 2000;60:7075-83.

17 Chia SK, Wykoff CC, Watson PH, et al. Prognostic significance of a novel hypoxia-regulated marker, carbonic anhydrase IX, in invasive breast carcinoma. J Clin Oncol 2001;19:3660-8.

18 Loncaster JA, Harris AL, Davidson SE, et al. Carbonic anhydrase (CA IX) expression, a potential new intrinsic marker of hypoxia: correlations with tumor oxygen measurements and prognosis in locally advanced carcinoma of the cervix. Cancer Res 2001;61:6394-9.

19 Giatromanolaki A, Koukourakis MI, Sivridis E, et al. Expression of hypoxiainducible carbonic anhydrase-9 relates to angiogenic pathways and independently to poor outcome in non-small cell lung cancer. Cancer Res $2001 ; 61: 7992-8$.

20 Olive PL, Aquino-Parsons C, MacPhail SH, et al. Carbonic anhydrase 9 as an endogenous marker for hypoxic cells in cervical cancer. Cancer Res 2001;61:8924-9.

21 Pastorekova S, Zavadova Z, Kostal M, et al. A novel quasi-viral agent, MaTu, is a two-component system. Virology 1992;187:620-6.

22 Colpaert C, Vermeulen $P$, van Beest $P$, et al. Intratumoral hypoxia resulting in the presence of a fibrotic focus is an independent predictor of early distant relapse in lymph node-negative breast cancer patients. Histopathology $2001 ; 39: 416-25$

23 Colpaert CG, Vermeulen PB, Fox SB, et al. The presence of a fibrotic focus in invasive breast carcinoma correlates with the expression of carbonic anhydrase IX and is a marker of hypoxia and poor prognosis. Breast Cancer Res Treat 2003;81:137-47.

24 Smith TA. Facilitative glucose transporter expression in human cancer tissue. Br J Biomed Sci 1999;56:285-92.

25 Chen C, Pore N, Behrooz A, et al. Regulation of glut1 mRNA by hypoxiainducible factor-1. Interaction between $\mathrm{H}$-ras and hypoxia. J Biol Chem $2001 ; 276: 9519-25$.

26 Michalides $\mathbf{R}$, van Tinteren $H$, Balkenende A, et al. Cyclin A is a prognostic indicator in early stage breast cancer with and without tamoxifen treatment. Br J Cancer 2002:86:402-8.

27 Elston CW, Ellis IO. Pathology and breast screening. Histopathology 1990;16:109-18.

28 Jubb AM, Pham TQ, Hanby AM, et al. Expression of vascular endothelial growth factor, hypoxia inducible factor $1 \alpha$, and carbonic anhydrase IX in human tumours. J Clin Pathol 2004:57:504-12.

29 Zhong H, De Marzo AM, Laughner E, et al. Overexpression of hypoxiainducible factor lalpha in common human cancers and their metastases. Cancer Res 1999;59:5830-5.

30 Tomes L, Emberley E, Niu Y, et al. Necrosis and hypoxia in invasive breast carcinoma. Breast Cancer Res Treat 2003:81:61-9.

31 Kaluz S, Kaluzova M, Chrastina A, et al. Lowered oxygen tension induces expression of the hypoxia marker MN/carbonic anhydrase IX in the absence of hypoxia-inducible factor 1 alpha stabilization: a role for phosphatidylinositol 3'-kinase. Cancer Res 2002;62:4469-77.

32 Rafajova M, Zatovicova M, Kettmann R, et al. Induction by hypoxia combined with low glucose or low bicarbonate and high posttranslational stability upon reoxygenation contribute to carbonic anhydrase IX expression in cancer cells. Int J Oncol 2004;24:995-1004.

33 Ramanujan S, Koenig GC, Padera TP, et al. Local imbalance of proangiogenic and antiangiogenic factors: a potential mechanism of focal necrosis and dormancy in tumors. Cancer Res 2000;60:1442-8.

34 Aebersold DM, Burri P, Beer KT, et al. Expression of hypoxia-inducible factorlalpha: a novel predictive and prognostic parameter in the radiotherapy of oropharyngeal cancer. Cancer Res 2001;61:2911-16.

35 Anastasiadis AG, Ghafar MA, Salomon L, et al. Human hormone-refractory prostate cancers can harbor mutations in the $\mathrm{O}(2)$-dependent degradation domain of hypoxia inducible factor-1 alpha (HIF-lalpha). J Cancer Res Clin Oncol 2002; 128:358-62

36 Saramaki OR, Savinainen KJ, Nupponen NN, et al. Amplification of hypoxiainducible factor lalpha gene in prostate cancer. Cancer Genet Cytogenet $2001 ; 128: 31-4$. 